\title{
Ethanol neurotoxicity is mediated by changes in expression, surface localization and functional properties of glutamate AMPA receptors
}

\author{
Elisabetta Gerace ${ }^{1}$ (D) | Alice llari ${ }^{1,2}$ | Lucia Caffino ${ }^{3}$ | Daniela Buonvicino ${ }^{4}$ | \\ Daniele Lana $^{4}$ (D) | Filippo Ugolini ${ }^{4}$ | Francesco Resta ${ }^{5,6}$ | Daniele Nosi ${ }^{7}$ | \\ Maria Grazia Giovannini ${ }^{4}$ | Roberto Ciccocioppo ${ }^{2}$ | Fabio Fumagalli ${ }^{3}$ (D) | \\ Domenico E. Pellegrini-Giampietro $^{4}$ | Alessio Masi ${ }^{1}$ | Guido Mannaioni ${ }^{1}$
}

${ }^{1}$ Department of Neuroscience, Psychology, Drug Research and Child Health (NeuroFarBa), Section of Pharmacology and Toxicology, University of Florence, Florence, Italy

${ }^{2}$ School of Pharmacy, Pharmacology Unit, University of Camerino, Camerino, Italy

${ }^{3}$ Department of Pharmacological and Biomolecular Sciences, Università degli Studi di Milano, Italy

${ }^{4}$ Department of Health Sciences, Section of Clinical Pharmacology and Oncology, University of Florence, Florence, Italy

${ }^{5}$ European Laboratory for Non-Linear Spectroscopy, Sesto Fiorentino, Italy

${ }^{6}$ Department of Physics and Astronomy, University of Florence, Florence, Italy

${ }^{7}$ Department of Experimental and Clinical Medicine, University of Florence, Florence, Italy

Correspondence

Elisabetta Gerace, Department of Neuroscience, Psychology, Drug Research and Child Health (NeuroFarBa), University of Florence, Viale G. Pieraccini 6, 50139

Florence, Italy.

Email: elisabetta.gerace@unifi.it

Funding information

Fondazione Cassa di Risparmio di Firenze;

University of Florence; Zardi-Gori

Foundation; MIUR Progetto Eccellenza

\begin{abstract}
Modifications in the subunit composition of AMPA receptors (AMPARs) have been linked to the transition from physiological to pathological conditions in a number of contexts, including EtOH-induced neurotoxicity. Previous work from our laboratory showed that EtOH withdrawal causes CA1 pyramidal cell death in organotypic hippocampal slices and changes in the expression of AMPARs. Here, we investigated whether changes in expression and function of AMPARs may be causal for EtOH-induced neurotoxicity. To this aim, we examined the subunit composition, localization and function of AMPARs in hippocampal slices exposed to EtOH by using western blotting, surface expression assay, confocal microscopy and electrophysiology. We found that EtOH withdrawal specifically increases GluA1 protein signal in total homogenates, but not in the post-synaptic density-enriched fraction. This is suggestive of overall increase and redistribution of AMPARs to the extrasynaptic compartment. At functional level, AMPA-induced calcium influx was unexpectedly reduced, whereas AMPA-induced current was enhanced in CA1 pyramidal neurons following EtOH withdrawal, suggesting that increased AMPAR expression may lead to cell death because of elevated excitability, and not for a direct contribution on calcium influx. Finally, the neurotoxicity caused by $\mathrm{EtOH}$ withdrawal was attenuated by the non-selective AMPAR antagonist 2,3-dioxo-6-nitro-1,2,3,4-tetrahydrobenzo[f] quinoxaline-7-sulfonamide disodium salt as well as by the selective antagonist of GluA2-lacking AMPARs 1-naphthyl acetyl spermine. We conclude that EtOH neurotoxicity involves changes in expression, surface localization and functional properties of AMPARs, and propose GluA2-lacking AMPARs as amenable specific targets for the development of neuroprotective drugs in $\mathrm{EtOH}$-withdrawal syndrome.
\end{abstract}

Abbreviations: AMPARs, $\alpha$-amino-3-hydroxy-5-methyl-4-isoxazolepropionic acid receptors; CpAMPARs, Ca ${ }^{2+}$-permeable AMPA receptors; EtOH, ethanol; GluA, AMPA receptor subunit; GRIP, glutamate receptor-interacting protein; NASPM, 1-naphthyl acetyl spermine; NBQX, 2,3-dioxo-6-nitro-1,2,3,4-tetrahydrobenzo[f]quinoxaline-7-sulfonamide disodium salt; PI, propidium iodide; PICK1, protein interacting with C kinase-1; PSD, postsynaptic density; REST, repressor element-1 (RE1) silencing transcription factor gene; SAP97, synapse-associated protein 97; TIFs, Triton insoluble fraction.

Elisabetta Gerace and Alice llari equally contributed to the manuscript. 
KEYWORDS

AMPA receptors, CA1 injury, ethanol withdrawal, organotypic hippocampal slices, scaffold

proteins

\section{1 | INTRODUCTION}

Ethanol (EtOH) abuse causes persistent structural and functional alterations in the brain by mechanisms that are not yet fully understood. Many studies have shown permanent impairment in synaptic function and neuronal damage caused by $\mathrm{EtOH}$ exposure and withdrawal in multiple neuronal areas, including the hippocampus, with both in vivo and in vitro models of neurotoxicity (Gerace et al., 2016, 2019; Läck et al., 2007; Marty \& Spigelman, 2012). AMPA receptors (AMPARs) mediate the fast component of excitatory neurotransmission, and the dynamic regulation of these receptors, both at transcriptional and posttranscriptional level, is a crucial factor in the transition from physiological to pathological conditions. The homeostasis of GluA1-2-containing AMPARs is controlled by several scaffold and adhesion complex proteins that capture, retain and promote surface expression of AMPARs, thus, regulating physiological neuronal functions and, in certain conditions, cell fate. AMPARs are localized mostly in the postsynaptic density and their trafficking is tightly regulated by interactions with scaffold molecules controlling the distribution of AMPARs in the synaptic, intracellular and extrasynaptic pools (Newpher \& Ehlers, 2008). Several AMPAR interacting proteins have been identified, including glutamate receptor-interacting protein (GRIP) and protein interacting with $\mathrm{C}$ kinase-1 (PICK1), which have been shown to specifically interact with GluA2 subunit (Daw et al., 2000; DeSouza et al., 2002; Perez et al., 2001; Seidenman et al., 2003), the transmembrane protein stargazin, which interacts directly with AMPAR (Chen et al., 2000) and Synapse-associated protein 97 (SAP97), which specifically binds GluA1 subunit (Howard et al., 2010; Leonard et al., 1998).

Great attention has been focused on the GluA2 subunit as a result of its profound effects on AMPAR assembly, trafficking and ionic selectivity. A relative decrease in GluA2 subunit increases calcium permeability of AMPARs, an event that has been linked to the transition from physiological to pathological conditions in a number of contexts. For example, one of the earliest biological manifestations of dementia in Alzheimer disease (AD) is owing to a reduction in synaptic AMPARs (Shankar et al., 2008). Moreover, prolonged decreases in surface GluA2-containing AMPARs have been described as a causal factor for cell death of hippocampal CA1 pyramidal neurons in models of toxicity (Anzai et al., 2003; Gerace et al., 2014) and in pathological conditions including global ischemia (PellegriniGiampietro, 1997). Interestingly, dysfunctional $Q / R$ editing in GluA2 occurs in the motor neurons of patients with amyotrophic lateral sclerosis (ALS, Hideyama \& Kwak, 2011). Calcium-permeable AMPARs (CpAMPARs) have also been demonstrated to play an essential role in substance use disorders (SUD) models, as exposure to substances lead to AMPAR molecular switch and formation of CpAMPARs (Mameli et al., 2011; Pascoli et al., 2014). For example, it was recently shown that cocaine-induced potentiation of VTA excitatory synapses is mediated by the insertion of CpAMPARs to the synaptic membranes (Mills et al., 2017). Moreover, cadherin adhesion complex proteins have been shown to contribute to the stabilization of AMPARs into the synaptic membranes (Saglietti et al., 2007; Tai et al., 2008) and may contribute to the susceptibility to cocaine addiction by the stabilization of GluA1-2-containing AMPARs into synaptic membranes (Mills et al., 2017).

A recent paper from our laboratory has demonstrated that $\mathrm{EtOH}$ withdrawal induces cell death in mature organotypic hippocampal slices and that glutamate receptors are mediators of $\mathrm{EtOH}$ withdrawal-induced toxicity (Gerace et al., 2019). In particular, we found that EtOH withdrawal increases the AMPA-mediated spontaneous excitatory postsynaptic currents (EPSCs) as well as the expression of GluA1 but not GluA2 AMPAR subunits, suggesting that the toxicity induced by $\mathrm{EtOH}$ withdrawal could be caused by the formation of GluA2-lacking, calcium-permeable AMPARs.

On the basis of these considerations, here we sought to investigate the role of GluA2-lacking AMPARs in the toxicity induced by EtOH withdrawal.

\section{2 | MATERIALS AND METHODS}

Male and female Wistar rat pups (94 animals, 7-9 days old, weight $16 \pm 3 \mathrm{~g}$ ) were obtained from Charles River. Animals were housed at $23 \pm 1^{\circ} \mathrm{C}$ under a $12 \mathrm{hr}$ light-dark cycle (lights on at 07:00) and were fed a standard laboratory diet with ad libitum access to water. The experimental protocols were approved by the Italian Ministry of Health (Aut. 176; 17E9C.N.VAS) and the European Communities Council Directive of 2010/63/EU.

The authors further attest that all efforts were made to minimize the number of animals used and their suffering, as reported in the Guidelines McGill Module-1. The present study was NOT pre-registered. NO subjects were excluded in the present study. No blinding for experiments was performed.

\section{1 | Materials}

Ethanol (EtOH, CAS No: 64-17-5), 1-Naphthylacetyl spermine trihydrochloride (NASPM, catalogue number: \#N193) and propidium iodide (PI, CAS No: 25535-16-4) were purchased from Sigma. Tissue culture reagents were obtained from Gibco-BRL and Sigma. 2,3-Dioxo-6-nitro-1,2,3,4-tetrahydrobenzo[f]quinoxaline7-sulfonamide disodium salt (NBQX, catalogue number: \#1044/1), tetrodotoxin Citrate (TTX, catalogue number: \#1069), DL-2-Amino5-phosphonopentanoic acid (APV, catalogue number: \#0105) and 
pictotoxin (catalogue number: \#1128) were purchased from Tocris. (R,S)-AMPA (CAS No: \#74341-63-2) was purchased from Abcam.

\section{2 | Preparation of rat organotypic hippocampal slice cultures}

Organotypic hippocampal slice cultures were prepared as previously reported (Gerace et al., 2012, 2015; Landucci et al., 2018). Briefly, after decapitation hippocampi were isolated and removed from the brains of Wistar rat pups, transverse slices $(420 \mu \mathrm{m})$ were prepared using a Mcllwain tissue chopper and then transferred onto $30-\mathrm{mm}$ diameter semi-porous membranes inserts (Millicell-CM, catalogue number: \#PICM03050; Millipore), which were placed in six well tissue culture plates containing $1.2-\mathrm{ml}$ medium per well. Slices were maintained at $37^{\circ} \mathrm{C}$ in an incubator in an atmosphere of humidified air and $5 \% \mathrm{CO}_{2}$ for 10 days. Before experiments all the slices were screened for viability by phase-contrast microscopy analysis; slices displaying signs of neurodegeneration were discarded from the study (exclusion criteria).

\section{3 | Ethanol exposure and Drug Treatment in organotypic hippocampal slices}

The experiments were conducted as previously described in Gerace et al. (2016) and Gerace et al. (2019). Briefly, hippocampal slice cultures were exposed for 7 days to $150 \mathrm{mM}$ of EtOH. The medium was changed every day adding EtOH to the fresh culture medium. For control slices, the medium was changed every day by adding fresh culture medium. After 7 days of $\mathrm{EtOH}$ treatment, some of the slices were incubated in $\mathrm{EtOH}$ fresh culture medium, $\mathrm{EtOH}$-free medium or in EtOH-free medium plus the non-selective AMPA antagonist NBQX $(10 \mu \mathrm{M})$ and the selective blocker of GluA2-lacking AMPARs NASPM $(10 \mu \mathrm{M})$ for $24 \mathrm{hr}$ before they were assessed for neuronal injury using PI fluorescence.

\subsection{Assessment of CA1 pyramidal cell injury}

PI $(5 \mu \mathrm{g} / \mathrm{ml})$ was added to the medium either at the end of the 7-day $\mathrm{EtOH}$ incubation period or $24 \mathrm{hr}$ after it was removed from the medium. Thirty minutes later, fluorescence was viewed using an inverted fluorescence microscope (Olympus IX-50; Solent Scientific) equipped with a xenon-arc lamp, a low-power objective $(4 \times)$ and a rhodamine filter. Images were digitized using a video image obtained by a CCD camera (Diagnostic Instruments Inc.) controlled by software (InCyt Im1 ${ }^{\mathrm{TM}}$; Intracellular Imaging Inc.) and subsequently analysed using the Image-Pro Plus morphometric analysis software (Media Cybernetics). In order to quantify cell death, the CA1 hippocampal subfield was identified and encompassed in a frame using the drawing function in the image software (ImageJ; NIH) and the optical density of PI fluorescence was detected. There was a linear correlation between CA1 PI fluorescence and the number of injured CA1 pyramidal cells as detected by morphological criteria (PellegriniGiampietro et al., 1999).

\subsection{Western blot analysis}

The experiments were conducted as previously described in Gerace et al. (2015) and Gerace et al. (2019). Cultured slices were washed with cold $0.01 \mathrm{M}$ PBS and dissolved in 1\% SDS. Total protein levels were quantified using the BCA (bicinchoninic acid) Protein Assay (catalogue number: \# 23,225) (Pierce). Forty $\mu$ g of proteins were resolved by electrophoresis on SDS-polyacrylamide gel and transferred onto nitrocellulose membranes using the transblot TURBO (Bio-Rad). Blots were probed overnight at $4^{\circ} \mathrm{C}$ with primary antibodies, all diluted 1:1,000. Immunodetection was performed with secondary antibodies conjugated to horseradish peroxidase. The reactive bands were detected using chemiluminescence (ECLplus; Euroclone). Quantitative analysis was performed using the QuantityOne analysis software (Bio-Rad).

\section{6 | Fluorescence immunohistochemistry and quantitative analysis}

At the end of experiments, the organotypic slices were harvested and fixed $\mathrm{O} / \mathrm{N}$ in ice-cold paraformaldehyde, $4 \%$ in PBS buffer. The day after, slices were rinsed in PSB and placed for at least 2 days in $18 \%$ sucrose in PBS. Immunostaining was performed with the freefloating method reported in Lana et al. (2014). Day 1: Organotypic hippocampal slices were placed in a multiwell and washed 3 times for $5 \mathrm{~min}$ in PBS-TX, then blocked for 60 min with BB containing $10 \%$ Normal Goat Serum. Slices were then incubated overnight at $4^{\circ} \mathrm{C}$ under slight agitation with a combination of two different primary antibodies (anti-NeuN (catalogue number: \#ABN78), 1:400; Millipore; GluA1-AMPA subunit (catalogue number: \#AGC-004), 1:100 or GluA2-AMPA subunit (catalogue number: \#AGC-005), 1:100 (Alomone Labs) dissolved in BB.

Day 2: Sections were incubated for $2 \mathrm{hr}$ at room temperature in the dark with AlexaFluor 488-conjugated donkey anti-rabbit IgG secondary antibody diluted in BB and then for $2 \mathrm{hr}$ at room temperature in the dark with AlexaFluor 488-conjugated donkey anti-rabbit IgG (catalogue number: \#A32790) secondary antibody plus AlexaFluor 555 goat anti-mouse (catalogue number: \#A-21422; Invitrogen, Thermo Fisher) both diluted 1:400 in BB. After three washings, slices were mounted onto gelatin-coated slides using Vectashield hard set mounting medium with DAPI (catalogue number: \#H-1200-10; Vector Laboratories).

Slices were observed under a LEICA TCS SP5 confocal laser scanning microscope (Leica Microsystems CMS $\mathrm{GmbH}$ ) equipped with a $63 \times$ objective ( $z$ step of $0.3 \mu \mathrm{m}$ ). Confocal scans were acquired keeping all parameters constant. Image analyses were conducted on z-stacks projections which contained the region of interest, corresponding to 
the CA1 area, using Image J (National Institute of Health, http://rsb. info.nih.gov/ij). Quantification of GluA1 and GluA2 AMPA subunit immunoreactivity in CA1 was performed separately using Image J. Forty consecutive $z$-scans $(0,3 \mu \mathrm{m}$ each, total $12 \mu \mathrm{m})$ were stacked, starting at $10 \mu \mathrm{m}$ inside the slice and an appropriate and constant threshold level was selected. Quantitation of immunofluorescence was then obtained from the ratio between positive pixels above threshold and total pixels in each region of interest.

\subsection{Real-time PCR for REST and GluA2 mRNA expression}

Real-time PCR was performed as previously reported in Llorente et al. (2015) and Lapucci et al. (2017). Total ribonucleic acid (RNA) was extracted using Trizol Reagent (catalogue number: \#15596018; Life Technologies). Any contaminating DNA in the RNA samples was removed by incubation with DNase (Sigma Aldrich). Total RNA concentrations were determined using a NanoDrop ND-3300 spectrophotometer (NanoDrop Technologies). Isolated RNA was maintained at $80^{\circ} \mathrm{C}$ until further processing. One $\mu \mathrm{g}$ of RNA was reverse transcribed using iScript (Bio-Rad). Real-Time PCR was performed in duplicate using Rotor-Gene 3,000 (Qiagen) and the Rotor-Gene TM SYBR® Green PCR Kit (Qiagen), the reactions were run at $95^{\circ} \mathrm{C}$ for $30 \mathrm{~s}, 95^{\circ} \mathrm{C}$ for $5 \mathrm{~s}$ and $60^{\circ} \mathrm{C}$ for $15 \mathrm{~s}$ for 45 cycles. As an internal control for normalization, PCR reactions were performed concurrently with the amplification of a reference gene, 18S ribosomal RNA (rRNA).

The following primers were used:

Forward

primer

GACGGAGAGCAAACCCAAGACCAG, Reverse primer $\left(5^{\prime}-3^{\prime}\right)$ CTTGAGCCATCTCCGAGGAGGGTTC:

GluA2: Forward primer (5'-3') CCAAGGACTCGGGAAGTAAGG, Reverse primer $\left(5^{\prime}-3^{\prime}\right)$ CCCCCGACAAGGATGTAGAA;

RNA 18S: Forward primer (5'-3') GATTAAGTGCCCTTTGTA, Reverse primer $\left(5^{\prime}-3^{\prime}\right)$ GATCCGAGGGCCTCACTAAAC.

\section{8 | Preparation of protein extracts and western blot analyses}

Proteins from hippocampal slices were extracted as previously described with minor modifications (Caffino et al., 2018; Fumagalli et al., 2008). Briefly, hippocampal slices were homogenized in a teflon-glass homogenizer in cold $0.32 \mathrm{M}$ sucrose buffer $\mathrm{pH} 7.4$ containing $1 \mathrm{mM} \mathrm{HEPES}, 1 \mathrm{mM} \mathrm{MgCl}$, $1 \mathrm{mM} \mathrm{NaHCO} 3$ and $0.1 \mathrm{mM} \mathrm{PMSF}$ in presence of commercial cocktails of protease (Roche) and phosphatase (Sigma-Aldrich) inhibitors and an aliquot of each homogenate was then sonicated. The remaining homogenate was centrifuged at $13,000 \mathrm{~g}$ for $15 \mathrm{~min}$ obtaining a pellet. This pellet was resuspended in a buffer containing $75 \mathrm{mM} \mathrm{KCl}$ and 1\% Triton X-100 and centrifuged at 100,000 $\mathrm{g}$ for $1 \mathrm{hr}$. The resulting pellet, referred as post-synaptic density (PSD) or Triton X-100 insoluble fraction (TIF), was homogenized in a glass-glass potter in 20 mM HEPES, protease and phosphatase inhibitors and stored at $-20^{\circ} \mathrm{C}$ in presence of glycerol $30 \%$. Total proteins have been measured in the total homogenate and in the TIF fraction according to the Bradford Protein Assay procedure (Bio-Rad Laboratories, Italy), using bovine serum albumin as calibration standard.

Equal amounts of proteins of the homogenate $(6 \mu \mathrm{g})$ and of TIF fraction $(5 \mu \mathrm{g})$ were run on criterion TGX precast gels (Bio-Rad Laboratories) under reducing conditions and electrophoretically transferred onto nitrocellulose membrane (Bio-Rad Laboratories). Blots were blocked $1 \mathrm{hr}$ at room temperature with I-Block solution (catalogue number: \# T2015, Life Technologies, Italia, Italy) in TBS $+0.1 \%$ Tween-20 buffer and incubated with antibodies against the proteins of interest.

The conditions of the primary antibodies were the following: anti N-Cadherin (catalogue number: \#13116, 1:1,000; Cell Signaling Technology); anti mGluR5 (1:2000, Chemicon, USA); anti GRIP (catalogue number: \#ABN27; 1:1,000; Sigma Aldrich); anti GluA1 (catalogue number: \#13185; 1:2000; Cell Signaling Technology); anti GluA2 (catalogue number: \#13607; 1:1,000; Cell Signaling Technology); anti SAP97 (catalogue number: \#ab2057181:1,000; Abcam); anti PSD-95 (catalogue number: \#2507; 1:4,000, Cell Signaling Technology), anti $\beta$-catenin (catalogue number: \# sc-7963; 1:1,000, Santa Cruz Biotechnology) and anti $\beta$-Actin (catalogue number: \# A5316; 1:10,000; Sigma-Aldrich). Results were standardized using $\beta$-actin as the control protein, which was detected by evaluating the band density at $43 \mathrm{kDa}$. Immunocomplexes were visualized by chemiluminescence using the Chemidoc MP Imaging System (BioRad Laboratories). Gels were run three times each and the results represent the average from three different western blots.

\section{9 | Electrophysiological recordings}

Whole-cell voltage-clamp recordings were performed in CA1 pyramidal neurons from organotypic hippocampal slices as previously described (Gerace et al., 2019). The slices were chronically treated with $150 \mathrm{mM}$ of $\mathrm{EtOH}$ or under control conditions, followed by 4-6 hr of EtOH withdrawal before recordings. A single slice was removed from the culture insert, placed in a flow chamber positioned under the microscope objective and continuously perfused with warm $\left(34-35^{\circ} \mathrm{C}\right)$ artificial Cerebrospinal Fluid (aCSF), composed of (in mM) $130 \mathrm{NaCl}, 3.5 \mathrm{KCl}, 1.25 \mathrm{NaH}_{2} \mathrm{PO}_{4}, 24 \mathrm{NaHCO}_{3}, 10$ glucose, $2 \mathrm{CaCl}_{2}$ and $1 \mathrm{MgSO}_{4}$ and saturated with a $95 \% \mathrm{O}_{2}+5 \% \mathrm{CO}_{2}$ gas mixture. Whole-cell pipettes were pulled from thin-walled borosilicate capillaries (Harvard Apparatus) with a vertical puller (Narishige PP830, Narishige International Limited) back-filled with the following intracellular solution (in $\mathrm{mM}$ ): $\mathrm{K}^{+}$Methanesulfonate (120), $\mathrm{KCl}$ (15), HEPES (10), EGTA (0.1), $\mathrm{MgCl}_{2}$ (2), $\mathrm{Na}_{2}$ PhosphoCreatine (5), $\mathrm{Na}_{2}$ GTP (0.3) and MgATP (2), resulting in a bath resistance of 3-5 $M \Omega$. For coupled recordings of electrical and fluorescent calcium signals, $0.1 \mathrm{mM}$ of Fluo 4 pentapotassium salt (Molecular Probes) was added. Signals were sampled at $10 \mathrm{kHz}$ and low-pass filtered at $3 \mathrm{kHz}$ with an Axon Multiclamp 700B (Molecular Devices, Sunnyvale, 
CA, USA). After establishing a Giga-Ohm seal, a whole-cell configuration was achieved by rupturing the neuronal membrane. Access resistance was monitored during voltage clamp recordings with brief test pulses (-10 mV, $500 \mathrm{~ms}$ ), throughout the experiment. All data were analysed using pCLAMP (Axon Instruments) and GraphPad Software (San Diego, CA). AMPA-mediated electrical and optical responses were studied by obtaining sequences of $30 \times 30$-s recording frames from individual CA1 neurons in whole-cell voltage clamp $\left(\mathrm{V}_{\text {HOLDING }}=-65 \mathrm{mV}\right)$ configuration. After the achievement of stable baselines, a mix of inhibitors was bath applied which included the voltage-dependent sodium channel blocker TTX (1 $\mu \mathrm{M})$, the NMDA receptor antagonist DL-2-Amino-5-phosphonopentanoic acid (APV, $50 \mu \mathrm{M})$ and the $\mathrm{GABA}_{\mathrm{A}}$ receptor antagonist picrotoxin (PTX, $\left.50 \mu \mathrm{M}\right)$. After $5 \mathrm{~min}, 0.3 \mu \mathrm{M}$ AMPA was applied, in presence of inhibitors, in order to record pharmacologically isolated cellular responses.

\subsection{0 | Microfluorometric determination of calcium responses}

AMPA-induced calcium responses were studied as described in Carbone et al. (2017) with some modifications. During electrophysiological recordings, fluorescence signal was collected from a square-shaped window comprising the cell body of the neuron under investigation loaded with $100 \mu \mathrm{M}$ of the high-affinity, nonratiometric calcium dye Fluo 4 pentapotassium salt (Molecular Probes). Fluorescence was elicited with a $488 \mathrm{~nm}$ LED and collected with a photomultiplier tube (PMT; Cairns Research) with a $10 \mathrm{kHz}$ sampling rate. LED excitation was triggered with the electrophysiological protocol and consisted of $1 \mathrm{~s}$ of LED ON at the end of each 30-s gap-free recording. Somatic calcium responses (SCRs) are reported as $\Delta \mathrm{F} / F_{0}$, where $F_{0}$ signal was the baseline emission of the loaded neuron at rest, and $\Delta \mathrm{F}$ was defined as $F_{\text {AMPA }}-F_{0}$ where $F_{\text {AMPA }}$ was taken at the end of AMPA application. Background fluorescence was obtained by measuring the emission of a Fluo 4-free area of the slice and subtracting the obtained value from $F_{0}$. Off-line analysis was performed with Clampfit 10 (Molecular Devices) and Origin 9.1.

\subsection{1 | Statistical analysis}

Data are presented as means \pm SEM of $n$ experiments from independent cell preparations. Statistical significance of differences between PI fluorescence intensities, immunostaining or Western blot optical densities was evaluated by performing one-way ANOVA followed by Tukey's w test for multiple comparisons. For electrophysiological

(a)

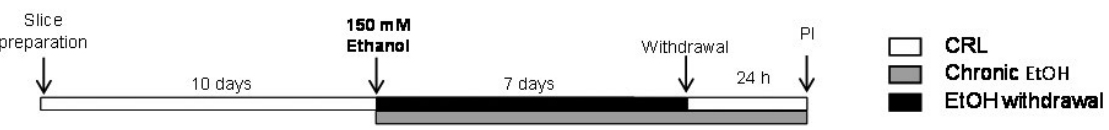

(b)
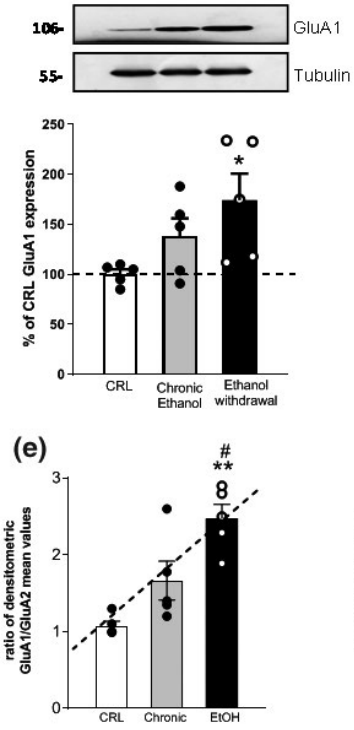

(c)

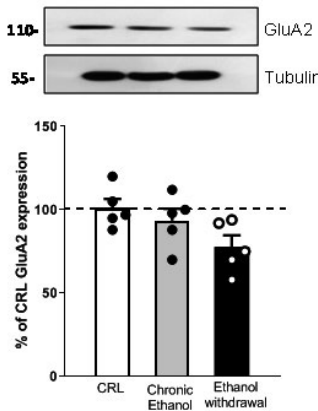

(f)

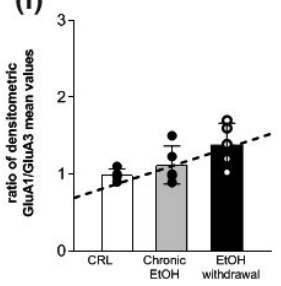

(d)

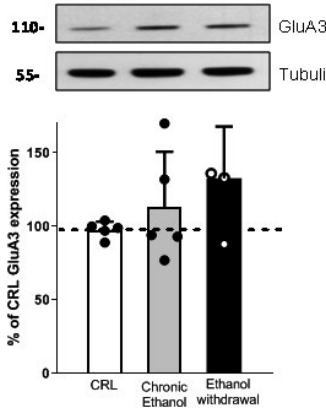

(g)

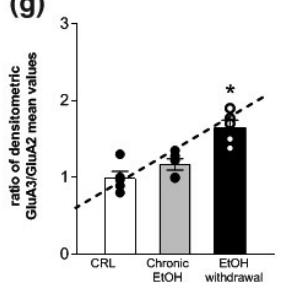

FIGURE 1 Ethanol withdrawal induces changes in $\alpha$-amino-3-hydroxy-5-methyl-4-isoxazolepropionic acid receptors (AMPARs) subunit composition. Top: experimental protocol (a). Middle: representative Western blots using antibodies directed against the AMPAR subunit GluA1, GluA2 and GluA3 (b, c, d) and quantitative analysis of immunoreactive bands (e, $f, g$ ), showing that EtOH withdrawal increases the expression of the AMPA subunit GluA1. Tubulin was used as loading control. Bottom: analysis of the ratio between GluA1/GluA2, GluA1/ GluA3 and GluA3/GluA2 AMPA subunits expression in mature slices after chronic EtOH and EtOH withdrawal suggesting that EtOH withdrawal modifies AMPA subunit composition leading to GluA2-lacking and $\mathrm{Ca}^{2+}$-permeable AMPARs. Data are expressed as a percentage of control. Bars represent the mean \pm SEMof at least five experiments from independent cell preparations (about $\geq 8$ slices for each experimental point). ${ }^{*} p<.05,{ }^{* *} p<0.1$ versus. CRL and ${ }^{\#} p<.05$ versus. Chronic EtOH (ANOVA + Tukey's test) 
experiments and determination of calcium response, statistical significance was evaluated by performing a Student's $t$ test for unpaired samples. Differences were considered significant for ${ }^{*} p<.05,{ }^{* *} p<.01$ and ${ }^{* * *} p<.001$. All statistical calculations were performed using GRAPH-PAD PRISM v.5 for Windows (GraphPad Software). No sample calculation was performed to predetermine the sample size. Data were not assessed for normality. NO test for outliers was conducted.

\section{3 | RESULTS}

We used rat organotypic hippocampal slice cultures exposed to $150 \mathrm{mM}$ EtOH for 7 days (chronic) to mimic chronic EtOH consumption and then we incubated the slices in $\mathrm{EtOH}$-free medium for the subsequent $24 \mathrm{hr}$ to mimic EtOH withdrawal in vitro. In these experimental conditions, EtOH withdrawal led to a dose-dependent CA1 (a)
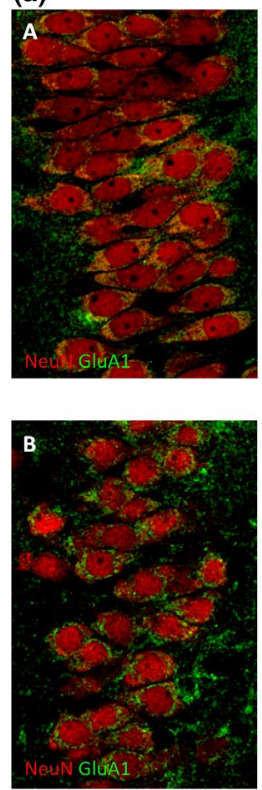

(b)
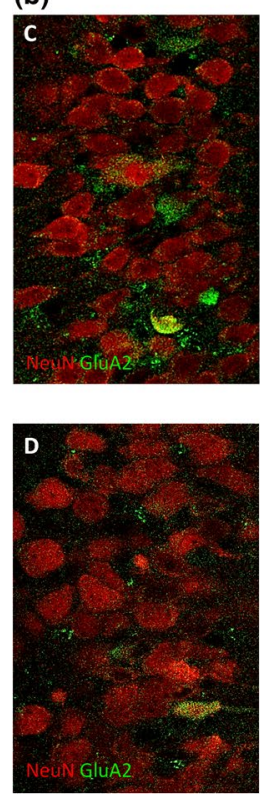

CTR

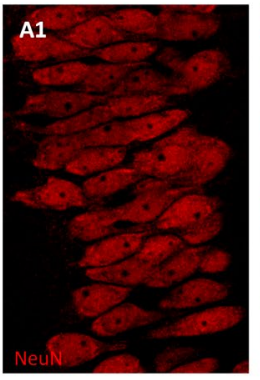

EtOH withdrawal

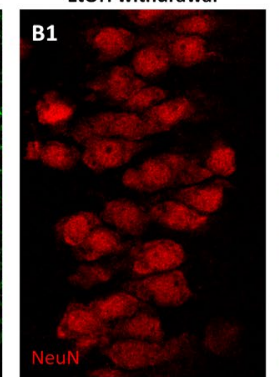

CTR

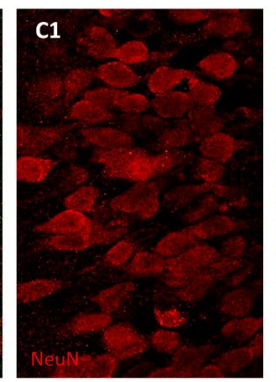

EtOH withdrawal

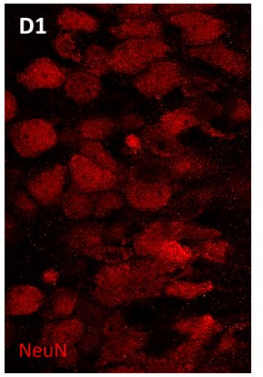

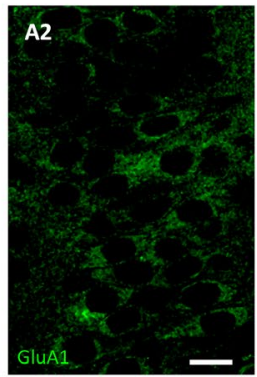
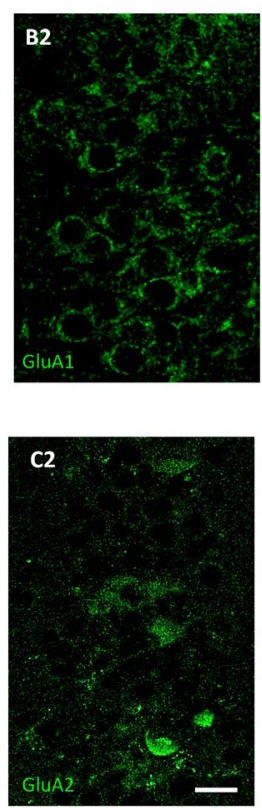

D2

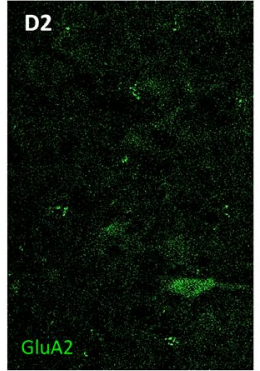

C
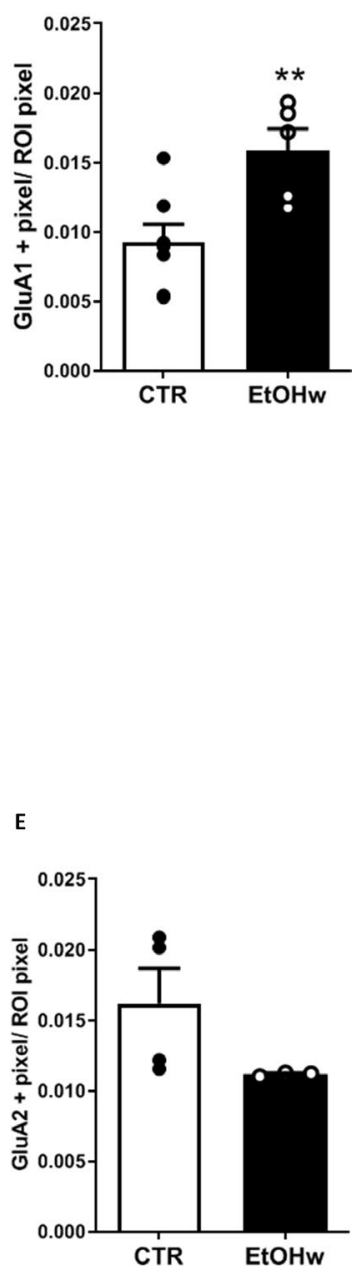

FIGURE 2 Ethanol withdrawal increases GluA1 AMPAR subunit expression in CA1 Stratum pyramidale of organotypic hippocampal slices. (a-b2) Representative confocal microscopy images showing CA1 immunostaining of neurons (NeuN, red, a1,b1), and GluA1AMPAR subunit (GluA1, green a2,b2) in CA1 pyramidal neurons of organotypic hippocampal slices in control (a-a2) or after 24h of EtOH withdrawal (b-b2). Scale bar: $10 \mu \mathrm{m}$. (c) Quantitative analysis of GluA1 immunoreactivity in CA1 Stratum pyramidale of control (CRL) (white column, $n=6$ ), and EtOH-withdrawal slices (black column, $n=6)\left({ }^{* *} p<.05 \mathrm{EtOH}\right.$ withdrawal versus. CRL, Student'sttest). (c-d2) Representative confocal microscopy images showing CA1 immunostaining of neurons (NeuN, red, c1,d1), and GluA2 AMPAR subunit (GluA2, green, c2, d2) in CA1 Stratum pyramidale of organotypic hippocampal slices in control (c-c2) or after 24h of EtOH withdrawal (d-d2). Scale bar: $10 \mu \mathrm{m}$. (e): Quantitative analysis of GluA2 immunoreactivity in CA1 Stratum pyramidale of control (CRL) (white column, $n=4)$, and EtOH-withdrawal slices (black column, $n=3$ ). The decrease was not statistically significant ${ }^{* *} p<0.1$ versus. CRL (Student's ttest) 
pyramidal cell injury examined using propidium iodide fluorescence as previously demonstrated and published (Gerace et al., 2016, 2019). With the aim to study the mechanism of EtOH-withdrawal toxicity, we firstly performed western blotting experiments to characterize the composition of AMPA receptor tetramers after EtOH withdrawal. We used specific antibodies directed against GluA1, GluA2 and GluA3 AMPAR subunits (Figure $1 \mathrm{a}-\mathrm{c}$ ). We found that $\mathrm{EtOH}$ withdrawal induced a significant increase in the expression of GluA1 (Figure 1a), no change was observed for GluA3 (Figure 1c), while a relative trend in a reduction was observed in the expression of GluA2 (Figure 1b) subunit. To this matter, the analysis of GluA1/GluA2 was significant after $\mathrm{EtOH}$ withdrawal as compared to control and to chronic EtOH and GluA3/GluA2 ratio expression was significant only versus control after EtOH withdrawal, while no changes was noticed for GluA1/ GluA3 ratio. These data suggest that $\mathrm{EtOH}$ withdrawal induces relevant modification of AMPAR subunit composition leading to formation of GluA2-lacking AMPARs (Figure 1d-f). We confirmed the data obtained by western blot analysis with immunofluorescence technique. Figure $2 a$ shows that GluA1 expression is significantly increased while GluA2 appears to be decreased (Figure 2b) in CA1 neurons after $24 \mathrm{~h}$ of $\mathrm{EtOH}$ withdrawal compared to control slices. In order to elucidate the mechanisms of GluA2 lacking AMPA formation, we considered another type of evidence resulting from the analysis of mRNA expression levels. In particular, we used real-time PCR to study the transcriptional repressor element-1 (RE1) silencing transcription factor (REST) gene, which was demonstrated to repress the transcription of GluA2 AMPAR subunit (Noh et al., 2012). The results showed that EtOH withdrawal induces the up-regulation of REST gene, conversely GluA2 mRNA gene was down-regulated (Figure 3), indicating that GluA2-lacking AMPARs formation could be caused by a transcriptional process. Furthermore, in order to study the trafficking of AMPARs, we measured the expression levels of GluA1 and GluA2 in enriched postsynaptic membranes (PSD, Figure S1) under control, chronic EtOH or EtOH withdrawal (Figure 4). In contrast with the previously shown increase in GluA1 subunit in total homogenates, these data showed that EtOH down-regulates the expression of both GluA1 and GluA2 AMPA subunits in PSD, suggesting that AMPARs should be relocated in the intracellular and/or extrasynaptic membrane compartments after $\mathrm{EtOH}$ treatment. Since cadherin adhesion complex proteins have been recently shown to contribute to the stabilization of GluA1/2-containing AMPARs into the synaptic membranes (Mameli et al., 2011; Mills et al., 2017), we analysed the levels of the scaffold proteins SAP97, GRIP and of N-cadherins in total homogenate and PSD preparations of organotypic slices under control, chronic EtOH or EtOH-withdrawal conditions (Figure 5). Our results showed that in total homogenate there is no modification of the expression level of the proteins examined in any experimental conditions. On the contrary, a clear reduction in their expression was present in PSD. Collectively, these results indicate that chronic $\mathrm{EtOH}$ induces profound alterations in the molecular composition and surface expression of AMPARs. We then sought evidence that $\mathrm{EtOH}$-induced molecular rearrangements result in increased calcium conductance through GluA2-lacking AMPARs. To directly test this hypothesis, we simultaneously measured electrophysiological and fluorescence calcium responses induced by AMPA stimulation in organotypic hippocampal slices during EtOH-withdrawal and in control conditions. These experiments were performed during early withdrawal (4-6 hr), when neurons are still viable and amenable to functional investigation. CA1 pyramidal neurons
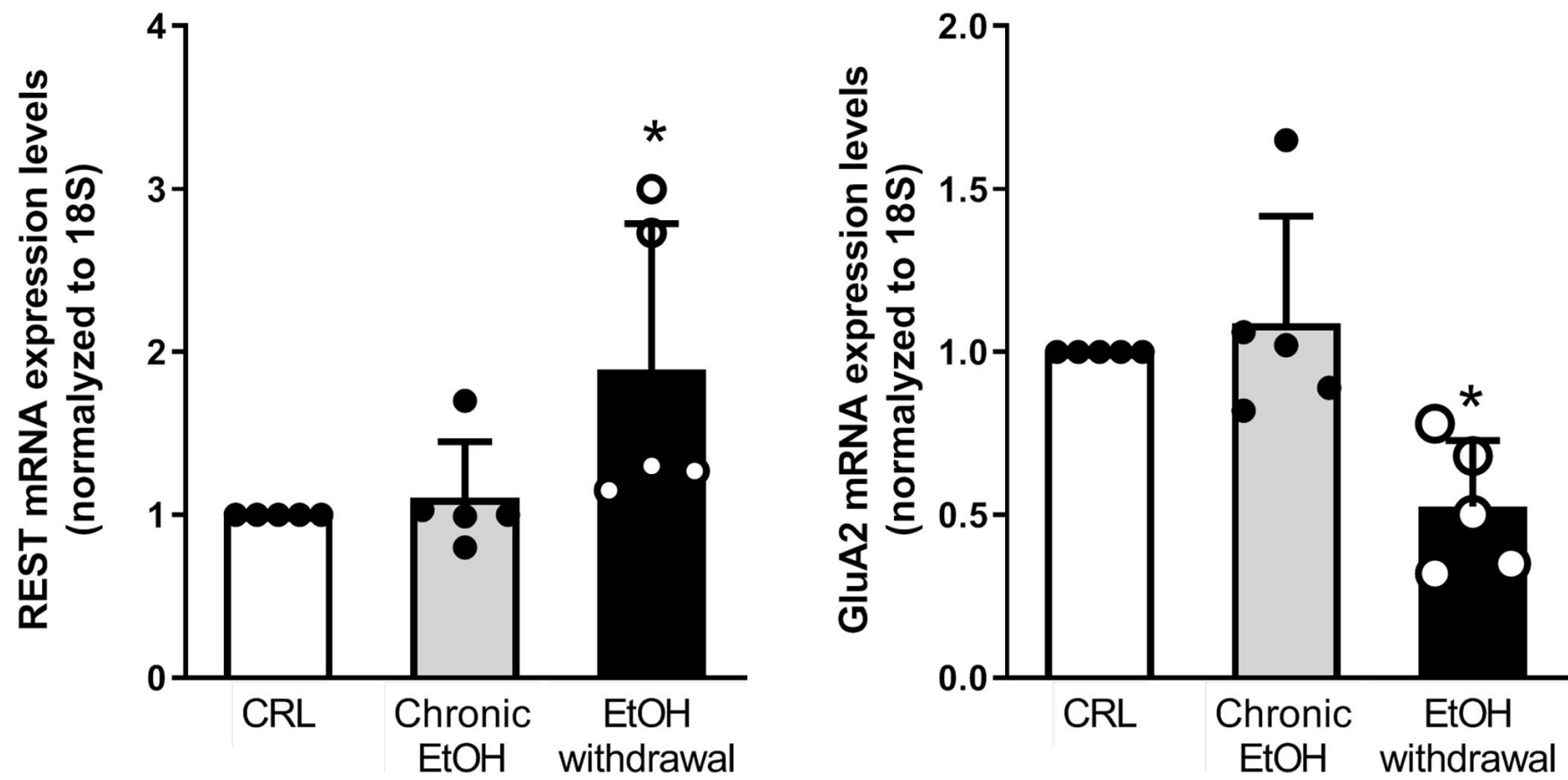

FIGURE 3 Ethanol withdrawal induces the up-regulation of the transcriptional repressor element-1 (RE1) silencing transcription factor (REST). Real-time PCR assessed mRNA expression levels of REST and of the AMPA subunit GluA2 gene after 24h of EtOH withdrawal. Data are expressed as percentage of control protein levels (white column). Bars represent the mean \pm SEMof four experiments from independent cell preparations (about $\geq 4$ slices for each experimental point). ${ }^{*} p<.05$ versus. CRL (ANOVA + Tukey's w test) 


\section{Postsynaptic density}
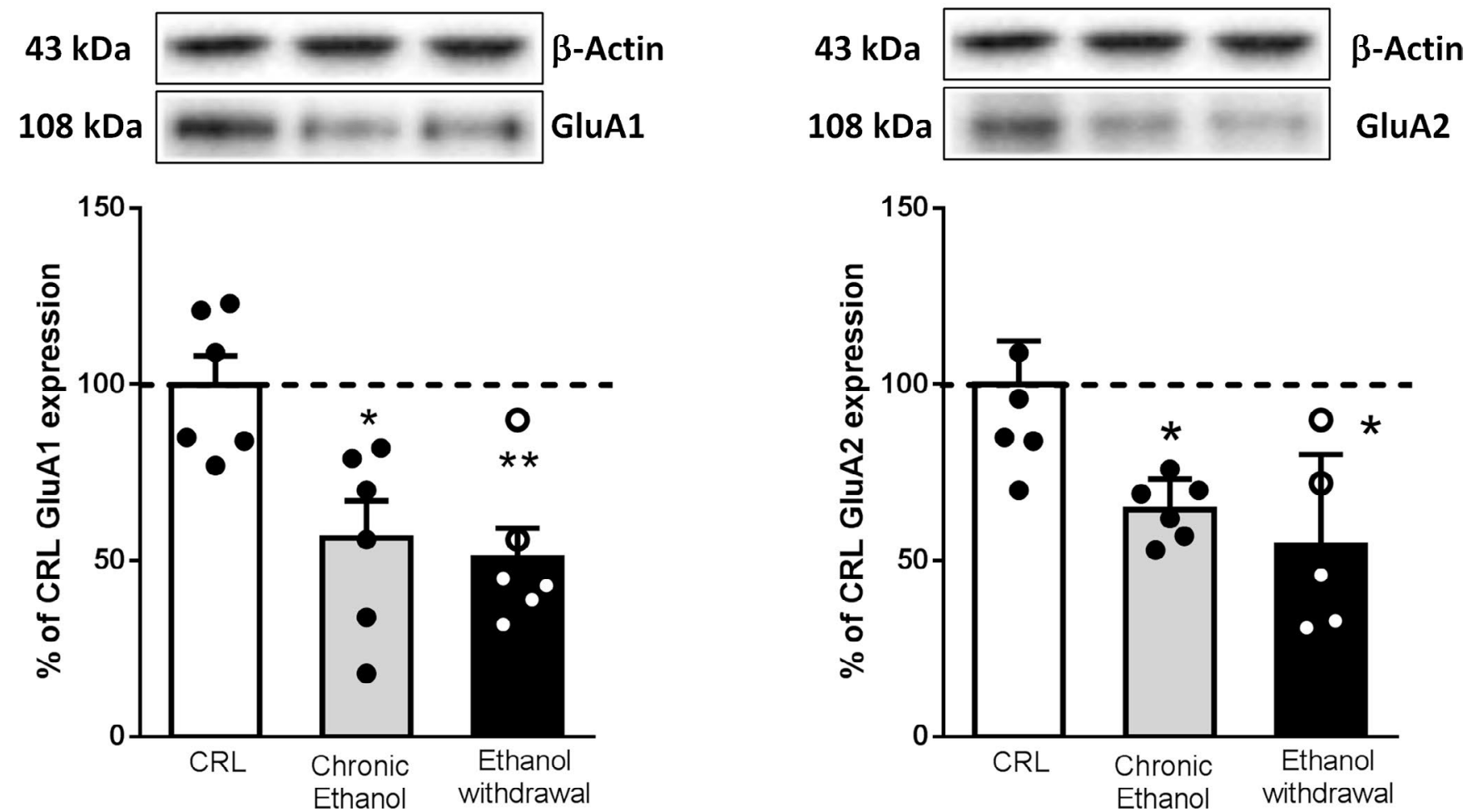

FIGURE 4 EtOH induces a significant reduction in GluA1 and GluA2 AMPA receptor subunit expression in the postsynaptic densities (PSD). The experiments were conducted as described in Figure 1. Representative immunoblots showing that the main AMPA receptor subunits GluA1 (left panel) and GluA2 (right panel) are significantly decreased after chronic EtOH and EtOH-withdrawal treatment in the PSD of organotypic hippocampal slices. Data are expressed as percentage of control protein levels (white column). Bar represent the mean \pm SEM of six experiments from independent cell preparations (about $\geq 12$ slices for each experimental point). ${ }^{* *} p<.01$ and ${ }^{*} p<.05$ versus. CRL (ANOVA + Tukey's w test)

were individually loaded with the non-ratiometric calcium dye Fluo4 and then AMPA-mediated electrical and fluorescence responses were elicited by agonist application. Our results show that AMPA reliably induces an inward cationic current in both controls and $\mathrm{EtOH}$-treated slices. Moreover, both peak value and area under the curve (AUC) of AMPA-induced inward currents are significantly increased in CA1 neuron after EtOH withdrawal compared to control slices (Figure 6). These findings confirm that the EtOH-dependent rearrangements in expression and localization of AMPARs that we observed lead to functional changes. Of note, the increase in AMPA current is reflective of an actual increase in current density, since no differences in whole-cell capacitance (a proxy for soma size) were observed between the two conditions (Figure S5). AMPA application also induced detectable elevations in intracellular calcium in both experimental groups but, quite unexpectedly, the magnitude of AMPA-induced calcium transients was significantly smaller in $\mathrm{CA} 1$ neurons from $\mathrm{EtOH}$ treated slices compared to controls (Figure 6d). Our data suggest that GluA2-lacking AMPARs may lead to excitotoxicity and neuronal death by driving network hyperexcitability rather than by a direct contribution of GluA2-lacking AMPARs-mediated calcium influx. To selectively assess the contribution of GluA2-lacking AMPARs in EtOH-induced hyperexcitation, we tested the neuroprotective efficacy of 1-naphthyl acetyl spermine (NASPM), a selective blocker of GluA2-lacking AMPARs, to protect CA1 neurons from EtOH-withdrawal toxicity, compared to NBQX (a non-selective AMPA antagonist). Our data showed that incubation of NASPM $(10 \mu \mathrm{M})$ during the $24 \mathrm{~h}$ of EtOH withdrawal significantly attenuated $\mathrm{EtOH}$ withdrawal-induced injury as well as NBQX, suggesting that the neurotoxicity induced by $\mathrm{EtOH}$ withdrawal is largely mediated by GluA2-lacking AMPARs (Figure 7).

\section{4 | DISCUSSION}

We have recently demonstrated that $\mathrm{EtOH}$ withdrawal induces $\mathrm{CA} 1$ pyramidal cell death in organotypic hippocampal slices. In our experimental condition, $\mathrm{EtOH}$ neurotoxicity was largely prevented by the non-selective AMPAR blocker NBQX and preceded by an increase in the amplitude of spontaneous EPSCs and in GluA1/GluA2 expression ratio, thus, pointing to GluA2-lacking AMPARs and to AMPARmediated calcium currents, as a possible death mechanism involved in EtOH toxicity (Gerace et al., 2019). Building on this hypothesis, we investigated the implication of AMPARs and, in particular of 
(b)

(a)

Homogenate Postsynaptic density

$130 \mathrm{kDa}$
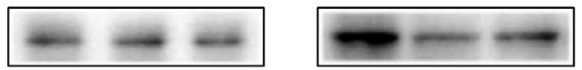

GRIP

$97 \mathrm{kDa}$
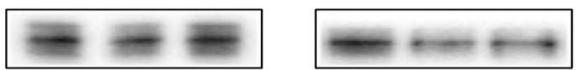

SAP97

$135 \mathrm{kDa}$
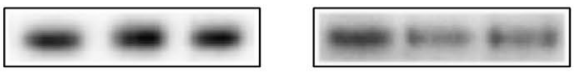

$\mathrm{N}$-Cadherin

$43 \mathrm{kDa}$
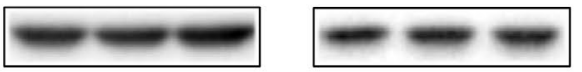

$\beta$-Actin
Homogenate
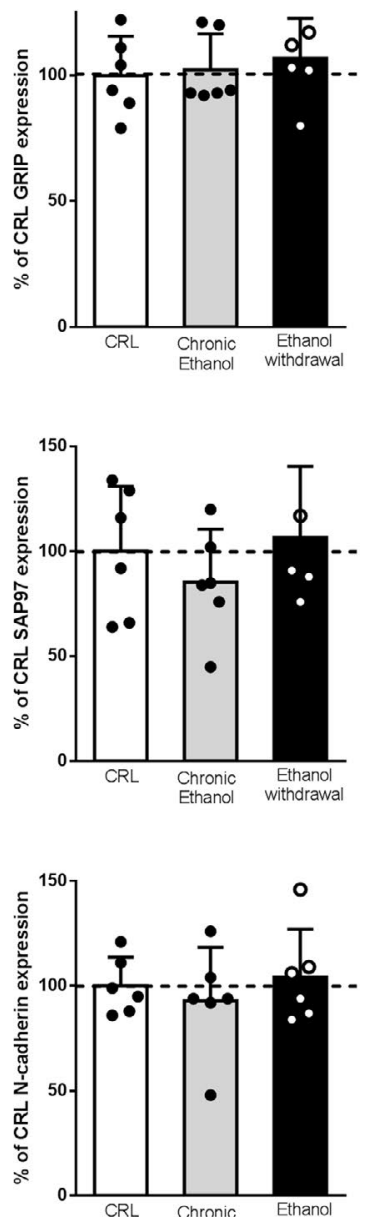

Postsynaptic density
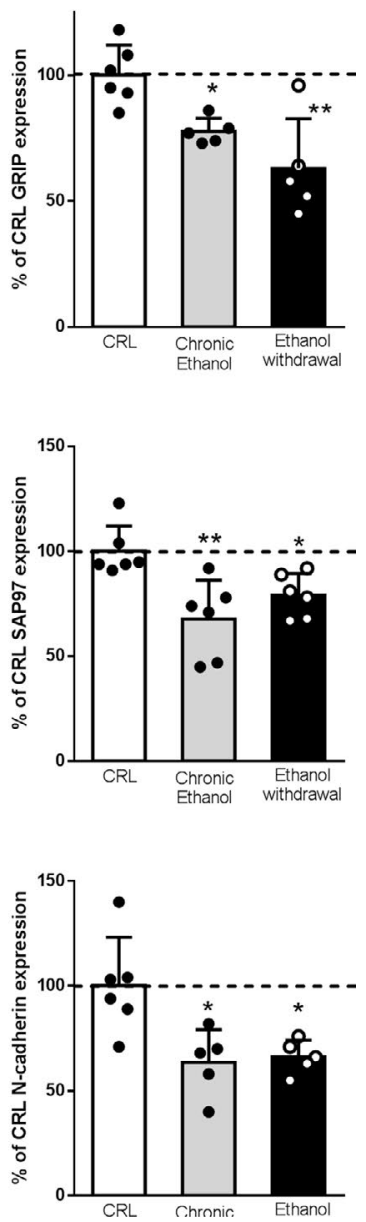

FIGURE 5 Effects of EtOH withdrawal on glutamate receptor-interacting protein (GRIP), synapse-associated protein 97 (SAP97) and $\mathrm{N}$-cadherin expression in the whole homogenate and post-synaptic densities (PSD). (a) Representative immunoblots showing that GRIP, SAP97 and N-cadherin proteins expression are significantly decreased after chronic EtOH and EtOH-withdrawal treatment in the PSD, but not in the homogenate of organotypic hippocampal slices. Panel (b) shows the protein levels of GRIP1 (upper panels), SAP97 (middle panels) and $\mathrm{N}$-cadherin (lower panel). Data are expressed as percentage of control protein levels (white column). Bar represent the mean \pm SEMof six experiments from independent cell preparations (about $\geq 12$ slices for each experimental point). ${ }^{* *} p<.01$ and ${ }^{*} p<.05$ versus. CRL (ANOVA + Tukey's w test)

GluA2-lacking AMPARs, in EtOH toxicity by using biochemistry, molecular biology, microscopy and physiology. We first examined the expression levels of the main AMPAR subunits (GluA1, GluA2, GluA3) in the total homogenate of hippocampal slices chronically exposed to EtOH for 7 days followed by 24-hr withdrawal. We found that $\mathrm{EtOH}$ withdrawal leads to a significant increase in overall GluA1, but not GluA2 and GluA3, expression, thus, pointing to an increase in the number of functional AMPARs (Figure 1b). Additionally, the significant increase in GluA1/GluA2 ratio suggests the molecular switch to GluA2-lacking AMPARs (Figure 1e), in line with previous works (Acosta et al., 2012; Jin et al., 2014; Li et al., 2017). Remarkably, the switch to GluA2-lacking AMPARs has been implicated in the alterations associated with EtOH consumption, such as behavioural reinforcement (Mameli et al., 2011; Marty \& Spigelman, 2012). In addition, it has been reported that repeated systemic administration of EtOH causes facilitation of LTP that is mediated by the insertion of AMPAR into the synaptic membrane and in a long-lasting increase in the GluA1 and GluA2 AMPAR subunits expression in the dorsomedial striatum of rats (Wang et al., 2012).

In the attempt to reveal the mechanism underlying the formation of GluA2-lacking AMPARs, as a consequence of EtOH withdrawal, we examined the mRNA expression of REST gene, one of the repressors involved in the regulation of the AMPAR subunit GluA2 (Noh et al., 2012). The activation of REST is clinically relevant in a model of ischemic stroke in vivo where it was reported to bind a subset of transcriptionally responsive genes, including GluA2 (Noh et al., 2012). Consistently, we found that REST gene expression is significantly up-regulated as a consequence of withdrawal and, at the same time, GluA2 gene expression is significantly reduced.

In order to get further insights into the modulation of AMPAR expression and function, we investigated the proteins regulating the trafficking and localization of these receptors to the post-synapse. 
(a)

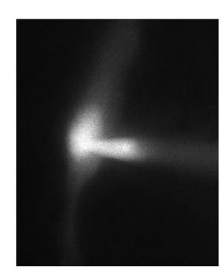

(b)
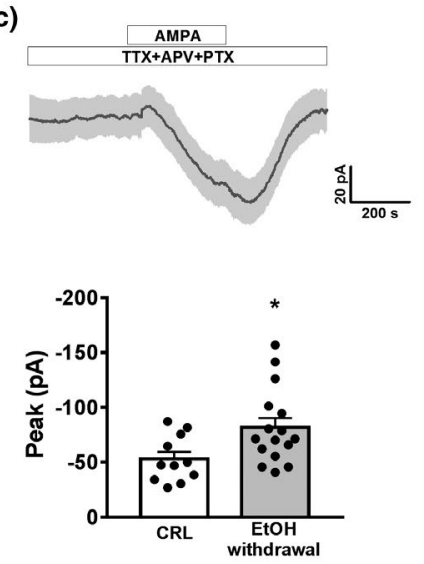

(d)
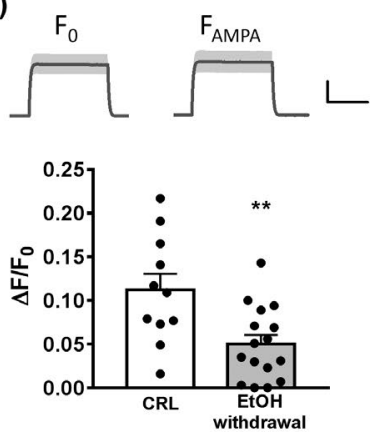

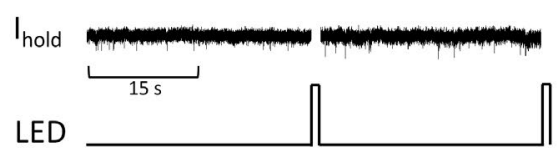

PMT
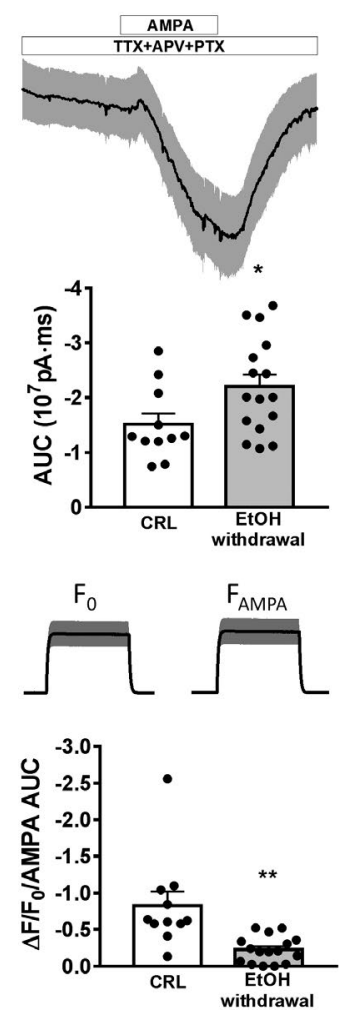

FIGURE 6 Electrophysiological and fluorescence calcium responses induced by AMPA stimulation in organotypic hippocampal slices. (a) CA1 pyramidal cell filled with Fluo-4 excited with blue LED light. (b) Experimental paradigm.Top: Electrophysiological protocol consisted of 30×, 30-s gap-free recordings. Middle and bottom: Optical protocol consisted of 1-s LED pulse and fluorescence collection with PMT at the end of each 30-s gap-free recording. (c)Top: time course of isolated AMPA current reported as average of " $n$ " superimposed recordings \pm SEM(gray shading) from CRL (left, $n=11$ ) and EtOH-withdrawal group (right, $n=16$ ). Bottom: quantification of AMPA-mediated current expressed as peak amplitude (left; CRL: $-53.11 \pm 6.38$ pA, $n=11$; EtOH withdrawal: $-81.82 \pm 8.58 \mathrm{pA}, n=16$ ) and AUC (right; CRL: $-1.51 \pm 0.20107 \mathrm{pA} \cdot \mathrm{ms}, n=11$; EtOH withdrawal: $-2.20 \pm 0.22$ $107 \mathrm{pA} \cdot \mathrm{ms}, n=16)$. (d)Top: averaged fluorescence responses to pulsed LED excitation before (FO) and after $\left(\mathrm{F}_{\text {AMPA }}\right)$ agonist application, from CRL (left, $n=10$ ) and EtOH-withdrawal group (right, $n=14$ ). Scale bar, $x=500 \mathrm{~ms}, y=0.2 \Delta \mathrm{F} / \mathrm{FO}$.Bottom: quantification of absolute $\Delta F / F 0$ (left, CRL: $0.11 \pm 0.02, n=11$; EtOH withdrawal: $0.05 \pm 0.01, n=16)$ and $\Delta F / F_{0}$ normalized to total AMPA current (right, CRL: $-0.83 \pm 0.19, n=11$; EtOH withdrawal: $-0.23 \pm 0.04, n=16)$. All histograms report data as mean values \pm SEMversus. CRL group. ${ }^{*} p<.05$ and ${ }^{* *} p<.01$ versus. CRL group (Student'st-test)

To this end, we examined the levels of GluA1 and GluA2, as well as scaffold proteins SAP97, GRIP, N-cadherin and beta catenin in total homogenate and PSD-enriched fractions from organotypic slices in control, chronic EtOH or EtOH withdrawal. We observed a clear reduction in the expression of all the proteins analysed (GRIP, SAP97, $\mathrm{N}$-cadherin) in the PSD preparation. Together with the previously shown increase in GluA1 subunit in total homogenates, this result suggests a re-localization of AMPARs to extrasynaptic compartments. The extrasynaptic distribution of AMPARs have particularly relevant consequences for synapse function and the lateral distribution of AMPARs within the PSD provides an effective mechanism for modulating synaptic strength (MacGillavry et al., 2011; Newpher \& Ehlers, 2008). For example, hippocampal neurons exposed to TNF $\alpha$ treatment display an increased surface level of AMPARs after $15 \mathrm{~min}$, the majority of which are relocalized to extrasynaptic sites. This mechanism has been proposed to contribute to excitotoxic neuronal death and is prevented by GluA2-lacking AMPAR antagonist NASPM (Leonoudakis et al., 2008), suggesting a role for AMPAR-mediated calcium in neurotoxicity. Functional determination of AMPA-induced calcium influx and whole-cell current during early EtOH withdrawal confirms that increased expression and subunit remodelling of AMPARs involve deep and complex changes at functional level. The most striking finding was that AMPA-induced, AMPAR-mediated calcium entry was reduced in $\mathrm{CA} 1$ neurons of EtOH-treated slices during early withdrawal. This result, which came unexpected, based on biochemical data, seems to exclude a direct involvement of AMPAR-mediated calcium inflow in EtOH-withdrawal neurotoxicity. The paradoxical reduction in AMPA-induced calcium influx in presence of increased GluA2-lacking AMPAR levels is possibly caused by post-translational modulation (Ivanova et al., 2020), and requires further investigation which goes beyond the scope of the present study. In contrast, we found that AMPA-induced whole-cell currents in CA1 neurons subject to EtOH withdrawal were enhanced. This is consistent with the increased GluA1 levels and EPSCs amplitude that we previously reported in this model following EtOH withdrawal (Gerace et al., 2019). Furthermore, early single-channel recordings reported larger unitary conductance of GluA2-lacking AMPARs in heterologous expression systems (Swanson et al., 1997).

In light of these results, we propose increased network excitability, promoted by simultaneous increase in AMPAR function and removal of $\mathrm{EtOH}$-mediated potentiation of inhibition, as the main mechanism of EtOH excitotoxicity, in agreement with the protective effect exerted by AMPAR blockers. In this regard, the evidence that the non-selective blocker NBQX and the selective GluA2-lacking AMPAR blocker NASPM show equal neuroprotective action suggests that, at molecular level, GluA2-lacking AMPARs are the main molecular entities accounting for the increased AMPA-induced current and thereby potentially represent a specific target for the development of neuroprotectants against the neurotoxic effects of EtOH withdrawal. 
(a)

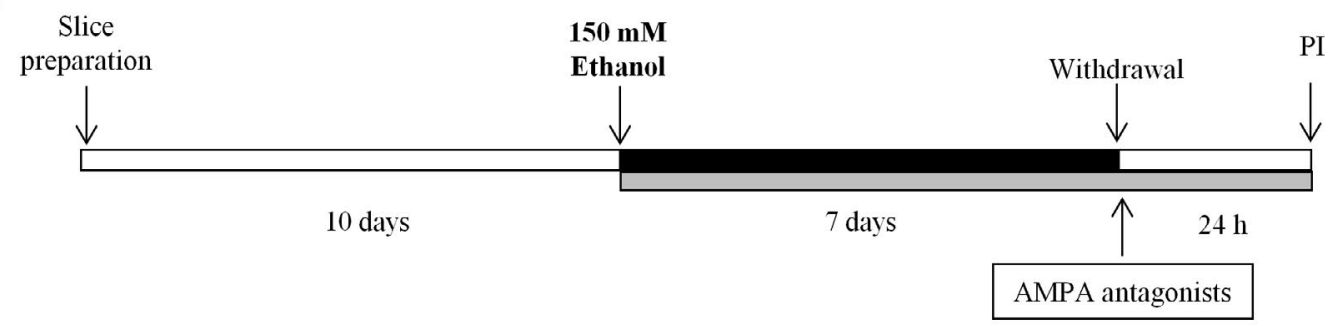

(b)

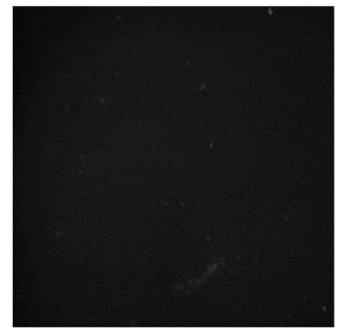

CRL

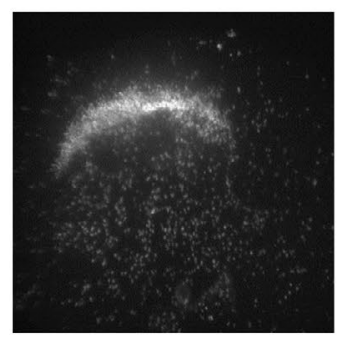

EtOH withdrawal $+$

NBQX

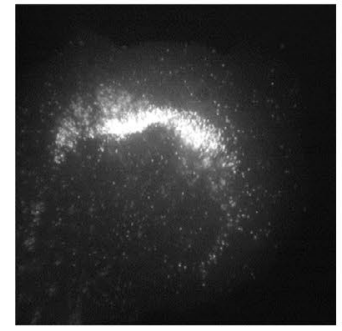

EtOH withdrawal

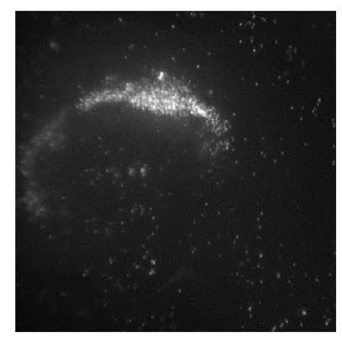

EtOH withdrawal

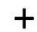

NASPM (c)

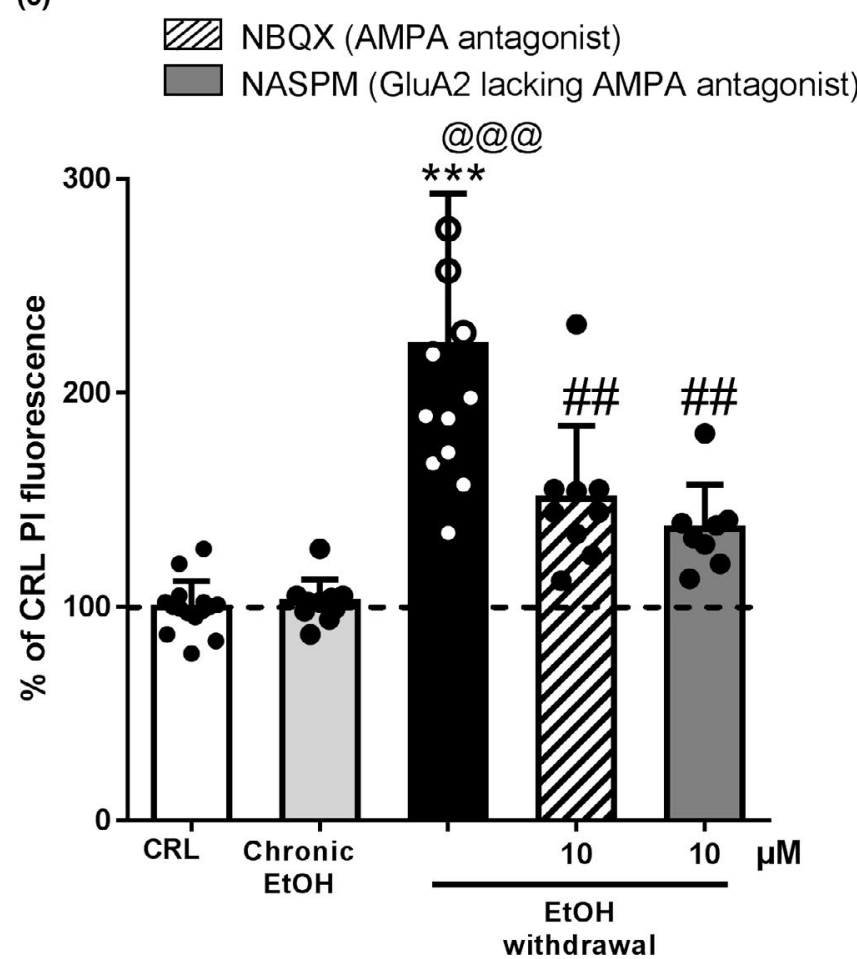

FIGURE 7 Neuroprotective effects of the selective GluA2-lacking $\alpha$-amino-3-hydroxy-5-methyl-4-isoxazolepropionic acid receptors (AMPARs) antagonist 1-naphthyl acetyl spermine (NASPM) on EtOH-withdrawal toxicity. (a) Experimental protocol. (b) Hippocampal slices displaying background levels of propidium iodide (PI) fluorescence under control conditions, an intense PI labelling in the CA1 subregion $24 \mathrm{hr}$ after EtOH withdrawal and a reduction in CA1 PI fluorescence when incubated with non-selective AMPA antagonist 2,3-dioxo-6-nitro-1,2,3,4-tetrahydrobenzo[f]quinoxaline-7-sulfonamide disodium salt (NBQX) and the selective blocker of GluA2lacking AMPARs NASPM. (c) Quantitative analysis of CA1 region expressed as percentage of CRL PI fluorescence. Values represent the mean \pm SEMof at least five experiments from independent cell preparations (about $\geq 8$ slices for each experimental point). ${ }^{* * *} p<.001$ versus. CRL Chronic EtOH, ${ }^{\# \#} p<.01 \mathrm{AMPA}$ antagonists versus. CRL and Chronic EtOH and ${ }^{@ @ p} p .001$ versus. EtOH withdrawal alone (ANOVA p Tukey's w test)

\section{ACKNOWLEDGMENTS}

This work was supported by grants from Fondazione Cassa di Risparmio di Firenze, University of Florence, Zardi-Gori Foundation and MIUR Progetto Eccellenza. The authors have no conflict of interest to declare.

All experiments were conducted in compliance with the ARRIVE guidelines.

\section{ORCID}

Elisabetta Gerace (iD https://orcid.org/0000-0001-8448-6092

Daniele Lana (iD https://orcid.org/0000-0002-5193-0019

Fabio Fumagalli iD https://orcid.org/0000-0002-8814-7706

\section{REFERENCES}

Acosta, G., Freidman, D. P., Grant, K. A., \& Hemby, S. E. (2012). Alternative splicing of AMPA subunits in prefrontal cortical fields of Cynomolgus Monkeys following chronic ethanol self-administration. Frontiers in Psychiatry, 2. https://doi.org/10.3389/ fpsyt.2011.00072

Anzai, T., Tsuzuki, K., Yamada, N., Hayashi, T., Iwakuma, M., Inada, K., Kameyama, K., Hoka, S., \& Saji, M. (2003). Overexpression of $\mathrm{Ca}^{2+}$ permeable AMPA receptor promotes delayed cell death of hippocampal CA1 neurons /following transient forebrain ischemia. Neuroscience Research, 46, 41-51.

Caffino, L., Messa, G., \& Fumagalli, F. (2018). A single cocaine administration alters dendritic spine morphology and impairs glutamate receptor synaptic retention in the medial prefrontal cortex of adolescent 
rats. Neuropharmacology, 140, 209-216. https://doi.org/10.1016/j. neuropharm.2018.08.006

Carbone, C., Costa, A., Provensi, G., Mannaioni, G., \& Masi, A. (2017). The hyperpolarization-activated current determines synaptic excitability, calcium activity and specific viability of Substantia Nigra dopaminergic neurons. Frontiers in Cellular Neuroscience, 28(11), 187. https://doi. org/10.3389/fncel.2017.00187

Chen, L., Chetkovich, D. M., Petralia, R. S., Sweeney, N. T., Kawasaki, Y., Wenthold, R. J., Bredt, D. S., \& Nicoll, R. A. (2000). Stargazin regulates synaptic targeting of AMPA receptors by two distinct mechanisms. Nature, 408, 936-943. https://doi.org/10.1038/35050030

Daw, M. I., Chittajallu, R., Bortolotto, Z. A., Dev, K. K., Duprat, F., Henley, J. M., Collingridge, G. L., \& Isaac, J. T. R. (2000). PDZ proteins interacting with C-terminal GluR2/3 are involved in a PKC-dependent regulation of AMPA receptors at hippocampal synapses. Neuron, 28, 873-886. https://doi.org/10.1016/S0896-6273(00)00160-4

DeSouza, S., Fu, J., States, B. A., \& Ziff, E. B. (2002). Differential palmitoylation directs the AMPA receptor-binding protein ABP to spines or to intracellular clusters. Journal of Neuroscience, 22, 3493-3503. https://doi.org/10.1523/JNEUROSCI.22-09-03493.2002

Fumagalli F., Frasca A., Racagni G. and Riva M. A. (2008) Dynamic Regulation of Glutamatergic Postsynaptic Activity in Rat Prefrontal Cortex by Repeated Administration of Antipsychotic Drugs. Mol Pharmacol 73, 1484-1490.

Gerace, E., Landucci, E., Bani, D., Moroni, F., Mannaioni, G., \& Pellegrini-Giampietro, D. E. (2019). Glutamate receptor-mediated neurotoxicity in a model of ethanol dependence and withdrawal in rat organotypic hippocampal slice cultures. Frontiers in Neuroscience, 12, 1053.

Gerace, E., Landucci, E., Scartabelli, T., Moroni, F., Chiarugi, A., \& Pellegrini-Giampietro, D. E. (2015). Interplay between histone acetylation/deacetylation and poly(ADP-ribosyl)ation in the development of ischemic tolerance in vitro. Neuropharmacology, 92, 125-134.

Gerace, E., Landucci, E., Scartabelli, T., Moroni, F., \& PellegriniGiampietro, D. E. (2012). Rat hippocampal slice culture models for the evaluation of neuroprotective agents. Methods in Molecular Biology, 846, 343-354.

Gerace, E., Landucci, E., Totti, A., Bani, D., Guasti, D., Baronti, R., Moroni, F., Mannaioni, G., \& Pellegrini-Giampietro, D. E. (2016). Ethanol toxicity during brain development: alterations of excitatory synaptic transmission in immature organotypic hippocampal slice cultures. Alcoholism, Clinical and Experimental Research, 40, 706-716. https:// doi.org/10.1111/acer.13006

Gerace, E., Masi, A., Resta, F., Felici, R., Landucci, E., Mello, T., PellegriniGiampietro, D. E., Mannaioni, G., \& Moroni, F. (2014). PARP-1 activation causes neuronal death in the hippocampal CA1 region by increasing the expression of $\mathrm{Ca}^{2+}$-permeable AMPA receptors. Neurobiology of Diseases, 70, 43-52. https://doi.org/10.1016/j.nbd.2014.05.023

Hideyama, T., \& Kwak, S. (2011). When Does ALS Start? ADAR2?GluA2 Hypothesis for the Etiology of Sporadic ALS. Frontiers in Molecular Neuroscience, 4, 33.

Howard, M. A., Elias, G. M., Elias, L. A. B., Swat, W., \& Nicoll, R. A. (2010). The role of SAP97 in synaptic glutamate receptor dynamics. Proceedings of the National Academy of Sciences, 107, 3805-3810.

Ivanova, V. O., Balaban, P. M., \& Bal, N. V. (2020). Modulation of AMPA receptors by nitric oxide in nerve cells. International Journal of Molecular Sciences, 21, 981. https://doi.org/10.3390/ijms21030981

Jin, Z., Bhandage, A. K., Bazov, I., Kononenko, O., Bakalkin, G., Korpi, E. R., \& Birnir, B. (2014). Selective increases of AMPA, NMDA, and kainate receptor subunit mRNAs in the hippocampus and orbitofrontal cortex but not in prefrontal cortex of human alcoholics. Frontiers in Cellular Neuroscience, 8, 11.

Läck, A. K., Diaz, M. R., Chappell, A., DuBois, D. W., \& McCool, B. A. (2007). Chronic ethanol and withdrawal differentially modulate pre- and postsynaptic function at glutamatergic synapses in rat basolateral amygdala. Journal of Neurophysiology, 98, 3185-3196. https://doi.org/10.1152/jn.00189.2007

Lana, D., Melani, A., Pugliese, A. M., Cipriani, S., Nosi, D., Pedata, F., \& Giovannini, M. G. (2014). The neuron-astrocyte-microglia triad in a rat model of chronic cerebral hypoperfusion: protective effect of dipyridamole. Frontiers in Aging Neuroscience, 6, 322.

Landucci, E., Filippi, L., Gerace, E., Catarzi, S., Guerrini, R., \& PellegriniGiampietro, D. E. (2018). Neuroprotective effects of topiramate and memantine in combination with hypothermia in hypoxic-ischemic brain injury in vitro and in vivo. Neuroscience Letters, 668, 103-107. https://doi.org/10.1016/j.neulet.2018.01.023

Lapucci, A., Cavone, L., Buonvicino, D., Felici, R., Gerace, E., Zwergel, C., Valente, S., Mai, A., \& Chiarugi, A. (2017). Effect of Class II HDAC inhibition on glutamate transporter expression and survival in SOD1ALS mice. Neuroscience Letters, 656, 120-125.

Leonard, A. S., Davare, M. A., Horne, M. C., Garner, C. C., \& Hell, J. W. (1998). SAP97 is associated with the $\alpha$-amino-3-hydroxy-5-methylisoxazole-4- propionic acid receptor GluR1 subunit. Journal of Biological Chemistry, 273, 19518-19524.

Leonoudakis, D., Zhao, P., \& Beattie, E. C. (2008). Rapid tumor necrosis factor -induced exocytosis of glutamate receptor 2-lacking AMPA receptors to extrasynaptic plasma membrane potentiates excitotoxicity. Journal of Neuroscience, 28, 2119-2130.

Li, J., Kang, S., Fu, R., Wu, L., Wu, W., Liu, H., Gregor, D., Zuo, W., Bekker, A., \& Ye, J.-H. (2017). Inhibition of AMPA receptor and CaMKII activity in the lateral habenula reduces depressive-like behavior and alcohol intake in rats. Neuropharmacology, 126, 108-120. https://doi. org/10.1016/j.neuropharm.2017.08.035

Llorente, I. L., Landucci, E., Pellegrini-Giampietro, D. E., \& FernándezLópez, A. (2015). Glutamate receptor and transporter modifications in rat organotypic hippocampal slice cultures exposed to oxygen-glucose deprivation: The contribution of cyclooxygenase-2. Neuroscience, 292, 118-128. https://doi.org/10.1016/j.neuroscience.2015.02.040

MacGillavry, H. D., Kerr, J. M., \& Blanpied, T. A. (2011). Lateral organization of the postsynaptic density. Molecular and Cellular Neurosciences, 48, 321-331.

Mameli, M., Bellone, C., Brown, M. T. C., \& Lüscher, C. (2011). Cocaine inverts rules for synaptic plasticity of glutamate transmission in the ventral tegmental area. Nature Neuroscience, 14, 414-416.

Marty, V. N., \& Spigelman, I. (2012). Long-lasting alterations in membrane properties, $\mathrm{K}^{+}$currents, and glutamatergic synaptic currents of nucleus accumbens medium spiny neurons in a rat model of alcohol dependence. Frontiers in Neuroscience, 6, 86.

Mills, F., Globa, A. K., Liu, S., Cowan, C. M., Mobasser, M., Phillips, A. G., Borgland, S. L., \& Bamji, S. X. (2017). Cadherins mediate cocaine-induced synaptic plasticity and behavioral conditioning. Nature Neuroscience, 20, 540-549.

Newpher, T. M., \& Ehlers, M. D. (2008). Glutamate receptor dynamics in dendritic microdomains. Neuron, 58, 472-497. https://doi. org/10.1016/j.neuron.2008.04.030

Noh, K.-M., Hwang, J.-Y., Follenzi, A., Athanasiadou, R., Miyawaki, T., Greally, J. M., Bennett, M. V. L., \& Zukin, R. S. (2012). Repressor element-1 silencing transcription factor (REST)-dependent epigenetic remodeling is critical to ischemia-induced neuronal death. Proceedings of the National Academy of Sciences, 109, E962-E971.

Pascoli, V., Terrier, J., Espallergues, J., Valjent, E., O'Connor, E. C., \& Lüscher, C. (2014). Contrasting forms of cocaine-evoked plasticity control components of relapse. Nature, 509, 459-464. https://doi. org/10.1038/nature13257

Pellegrini-Giampietro, D. (1997). The GluR2 (GluR-B) hypothesis: $\mathrm{Ca}^{2+}$-permeable AMPA receptors in neurological disorders. Trends in Neurosciences, 20, 464-470. https://doi.org/10.1016/S0166 $-2236(97) 01100-4$

Pellegrini-Giampietro, D. E., Peruginelli, F., Meli, E., Cozzi, A., AlbaniTorregrossa, S., Pellicciari, R., \& Moroni, F. (1999). Protection 
with metabotropic glutamate 1 receptor antagonists in models of ischemic neuronal death: Time-course and mechanisms. Neuropharmacology, 38, 1607-1619. https://doi.org/10.1016/ S0028-3908(99)00097-0

Perez, J. L., Khatri, L., Chang, C., Srivastava, S., Osten, P., \& Ziff, E. B. (2001). PICK1 targets activated protein kinase $\mathrm{C} \alpha$ to AMPA receptor clusters in spines of hippocampal neurons and reduces surface levels of the AMPA-type glutamate receptor subunit 2. Journal of Neuroscience, 21, 5417-5428. https://doi.org/10.1523/JNEUROSCI.21-15-05417.2001

Saglietti, L., Dequidt, C., Kamieniarz, K., Rousset, M.-C., Valnegri, P., Thoumine, O., Beretta, F., Fagni, L., Choquet, D., Sala, C., Sheng, M., \& Passafaro, M. (2007). Extracellular interactions between GluR2 and $\mathrm{N}$-cadherin in spine regulation. Neuron, 54, 461-477. https://doi. org/10.1016/j.neuron.2007.04.012

Seidenman, K. J., Steinberg, J. P., Huganir, R., \& Malinow, R. (2003). Glutamate receptor subunit 2 serine 880 phosphorylation modulates synaptic transmission and mediates plasticity in CA1 pyramidal cells. Journal of Neuroscience, 23, 9220-9228. https://doi.org/10.1523/ JNEUROSCI.23-27-09220.2003

Shankar, G. M., Li, S., Mehta, T. H., Garcia-Munoz, A., Shepardson, N. E., Smith, I., Brett, F. M. et al (2008). Amyloid- $\beta$ protein dimers isolated directly from Alzheimer's brains impair synaptic plasticity and memory. Nature Medicine, 14, 837-842.

Swanson, G. T., Kamboj, S. K., \& Cull-Candy, S. G. (1997). Singlechannel properties of recombinant AMPA receptors depend on RNA editing, splice variation, and subunit composition. Journal of Neuroscience, 17(1), 58-69. https://doi.org/10.1523/JNEUR OSCI.17-01-00058.1997

Tai, C.-Y., Kim, S. A., \& Schuman, E. M. (2008). Cadherins and synaptic plasticity. Current Opinion in Cell Biology, 20, 567-575.

Wang, J., Hamida, S., Ben, D. E., Zhu, W., Gibb, S. L., Lanfranco, M. F., Carnicella, S., \& Ron, D. (2012). Ethanol-mediated facilitation of AMPA receptor function in the dorsomedial striatum: Implications for alcohol drinking behavior. Journal of Neuroscience, 32, 1512415132. https://doi.org/10.1523/JNEUROSCI.2783-12.2012

\section{SUPPORTING INFORMATION}

Additional supporting information may be found online in the Supporting Information section.

How to cite this article: Gerace E, Ilari A, Caffino L, et al. Ethanol neurotoxicity is mediated by changes in expression, surface localization and functional properties of glutamate AMPA receptors. J Neurochem. 2020;00:1-13. https://doi. org/10.1111/jnc.15223 